\title{
The physics of liquid Para-Hydrogen
}

\author{
Thomas Lindenau, Manfred L. Ristig, ${ }^{1}$ Klaus A. \\ Gernoth, ${ }^{2}$ Javier Dawidowski, ${ }^{3}$ and Francisco J. Bermejo ${ }^{4}$ \\ ${ }^{1}$ Institut für Theoretische Physik, Universität zu Köln \\ D-50937 Köln, Germany \\ ristig@thp.uni-koeln.de \\ ${ }^{2}$ School of Physics and Astronomy, The University of Manchester \\ Manchester, United Kingdom \\ k.a.gernoth@man.ac.uk \\ ${ }^{3}$ Consejo Nacional de Investigationes Cientificas y Tecnicas, \\ Centro Atomico Bariloche and Instituto Balseiro, \\ Comision Nacional de Energia Atomica, \\ Universidad Nacional de Cuyo, (8400) Bariloche, Argentina \\ javier@cab.cnea.gov.ar \\ ${ }^{4}$ C.S.I.C. Dept. of Electricity and Electronics, \\ University of the Basque Country, P.O. 644, E-48080 Bilbao, Spain \\ javier@langran.iem.csic.es
}

\begin{abstract}
Macroscopic systems of hydrogen molecules exhibit a rich thermodynamic phase behavior. Due to the simplicity of the molecular constituents a detailed exploration of the thermal properties of these boson systems at low temperatures is of fundamental interest. Here,we report theoretical and experimental results on various spatial correlation functions and corresponding distributions in momentum space of liquid para-hydrogen close to the triple point. They characterize the structure of the correlated liquid and provide information on quantum effects present in this Bose fluid. Numerical calculations employ Correlated Density-Matrix(CDM)theory and Path-Integral MonteCarlo(PIMC)simulations. A comparison of these theoretical results demonstrates the accuracy of CDM theory. This algorithm therefore permits a fast and efficient quantitative analysis of the normal phase of liquid para-hydrogen. We compare and discuss the theoretical results with available experimental data.
\end{abstract}




\section{INTRODUCTION}

Microscopic, mesoscopic, and macroscopic systems of hydrogen atoms or molecules are of fundamental importance in quantum many-body theory. The properties of a single hydrogen atom led the basis for the atomic shell model. A single hydrogen molecule is the simplest compound and has been an early laboratory for studying chemical binding. Its spectrum exhibits the effects of internal degrees of freedom leading to different levels of excitations and the existence of ortho- and para-hydrogen. The molecule therefore shares common features with nuclei where internal degrees are important and cause violation of the so-called Ysymmetry ${ }^{1,2}$. Atomic hydrogen gas in a strong magnetic field remains a Bose fluid even at zero temperature and can condense into a Bose-Einstein phase under certain conditions $\mathrm{s}^{\underline{3}}$ like the alkali gases ${ }^{4}$.

Liquid and solid phases of molecular hydrogen and deuterium have been extensively studied, experimentally as well as theoretically. These many-body systems are of continuing interest for a variety of reasons. Hydrogen molecules are the dominant constituents of giant planets ${ }^{5}$. The physics of metallic hydrogen is explored by many researchers to achieve the insulator-metal cross-over ${ }^{6}$.

The hydrogen liquid, close to the triple point, is the object of current research. In this paper we concentrate on a theoretical and experimental analysis of its quantum properties close to the triple point. There are many other open and interesting questions, such as properties of mesoscopic clusters and films, molecular hydrogen in confined geometries, BoseEinstein condensation in solid and supercooled liquid hydrogen, etc.

We investigate the properties of the one- and two-body reduced density-matrix elements of liquid para-hydrogen in its normal boson phase at low temperatures.The associated Fourier transverse of these quantities reveal the spatial structure of the correlated system. Detailed numerical calculations are performed at the temperature $T=16 \mathrm{~K}$ and a particle number density $\rho=0.021 \AA^{-3}$. The experimental measurements for the liquid structure function ${ }^{7}$ and the dispersion law of the collective excitations ${ }^{8}$ have been done at the temperature $T=15.2 \mathrm{~K}$. Quantitative information on single-particle properties have been gained by precision neutron-scattering experiments at $T=15.7 \mathrm{~K}$ and various pressures that permits to extract the momentum distribution of a molecule in the hydrogen liquid ${ }^{9}$.

The present theoretical analysis is based on the parameter-free microscopic CDM 
theory ${ }^{10,11,12}$ and PIMC calculations ${ }^{13}$ with the central Silvera-Goldman potential as input ${ }^{14}$. Section 2 begins with a quantitative study of the spatial distribution function $g(r)$ and the static structure function $S(k)$ comparing the CDM results with the corresponding PIMC results. Theoretical results on the excitation energies and quasiparticle energies within CDM theory are discussed and compared with the measured dispersion laws in Section 3. The theoretical momentum distribution $n(k)$ is analysed in Section 4. Its study is based on a structural factorization of the one-body reduced density-matrix elements $n(r)$ reported earlier $^{15}$ that permits a clean separation of particle exchange properties from spatial phasephase correlations caused by the intermolecular interactions. A short summary is given in Section 5.

\section{SPATIAL STRUCTURE}

The spatial structure of a homogeneous quantum fluid in thermal equilibrium may be characterized by a set of correlation functions and concomitant Fourier transforms or structure functions. The radial distribution function $g(r)$ describes the spatial correlations between two particles in coordinate space and depends on the relative distance between them, $r=\left|\mathbf{r}_{1}-\mathbf{r}_{2}\right|$. The associated static structure function $S(k)$ is the dimensionless Fourier transform of $g(r)$.Its dependence on the relative momentum or wavenumber $\mathrm{k}$ can be extracted from measured neutron scattering cross sections. The quantum-mechanical correlations between identical particles become apparent in the cyclic or particle-exchange correlation function $G_{c c}(r)$ and its Fourier inverse $S_{c c}(k)$. Information on the spatial correlations between

the phase factors associated with the single-particle wave components of the full N-body density matrix of the thermodynamic state of the quantum fluid is embodied in the phasephase correlation function $Q(r)$. This quantity is contained in the off-diagonal elements $n(r)$, i.e., the one-body reduced density matrix.

This Section deals with a thorough analysis and comparison of theoretical and experimental results on functions $g(r)$ and $S(k)$. CDM theory provides a renormalized Schrödinger equation with zero eigenvalue for the square root of the radial distribution function,

$$
\left[-\frac{\hbar^{2}}{m} \Delta+v(r)+w(r)+v_{\text {coll }}(r)+v_{q p}(r)\right] \sqrt{g(r)}=0 .
$$

The potential energy terms appearing in Eq. (11) are the Silvera potential14 $v(r)$, the in- 


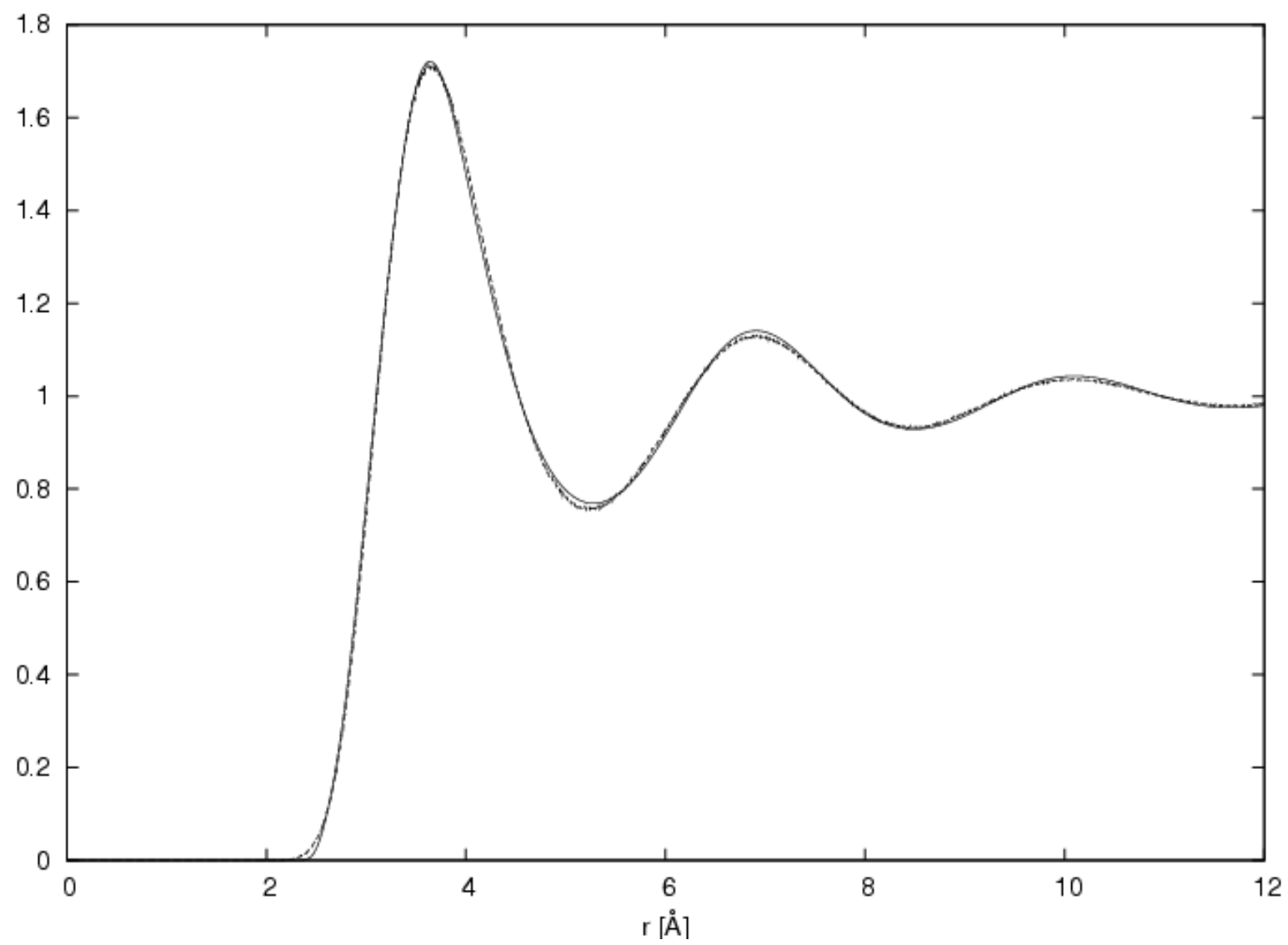

FIG. 1: Radial distribution function $g(r)$ of liquid para-hydrogen $\left(T=16 \mathrm{~K}, \rho=0.021 \AA^{-3}\right)$. Full line: results of CDM theory, broken line: PIMC simulation results.

duced potential ${ }^{12} w(r)$, the coupling term $v_{\text {coll }}(r)$ to the collective excitations $\frac{10}{}$, and the quasiparticle coupling term $\frac{12}{\underline{2}} v_{q p}(r)$. For liquid para-hydrogen close to the triple point the last coupling term is very small and may be ignored. Without the term $v_{q p}(r)$ it is straightforward to solve the Schrödinger equation by following the elementary calculus designed in Ref. 10. The solution is displayed in Figure 1. We have checked its numerical accuracy by comparing the CDM results with those of a PIMC calculation for the same temperature and density $\underline{16}$, finding excellent agreement(see Figure 1).

The results on the distribution $g(r)$ show the enormous correlation strength that exists in the hydrogen liquid. The first maximum at $r=3.7 \AA$ attains a value that is significantly larger than the maximal strength one finds at the first peak of the radial distribution function of liquid helium under similar thermal conditions assuming the realistic Aziz potentialli ${ }^{17}$ as input for the theoretical analysis (Figure 2). 
Next, we turn to a detailed study of the associated static structure function $S(k)$. Within CDM theory as well as in the stochastic PIMC approach this quantity is calculated as the dimensionless Fourier transform

$$
S(k)=1+\rho \int[g(r)-1] \mathrm{e}^{i \mathbf{k r}} d \mathbf{r} .
$$

Numerical results on the structure function with CDM and PIMC data for $g(r)$ as input are displayed in Figure 3. Once more, the Monte-Carlo results confirm the high accuracy of

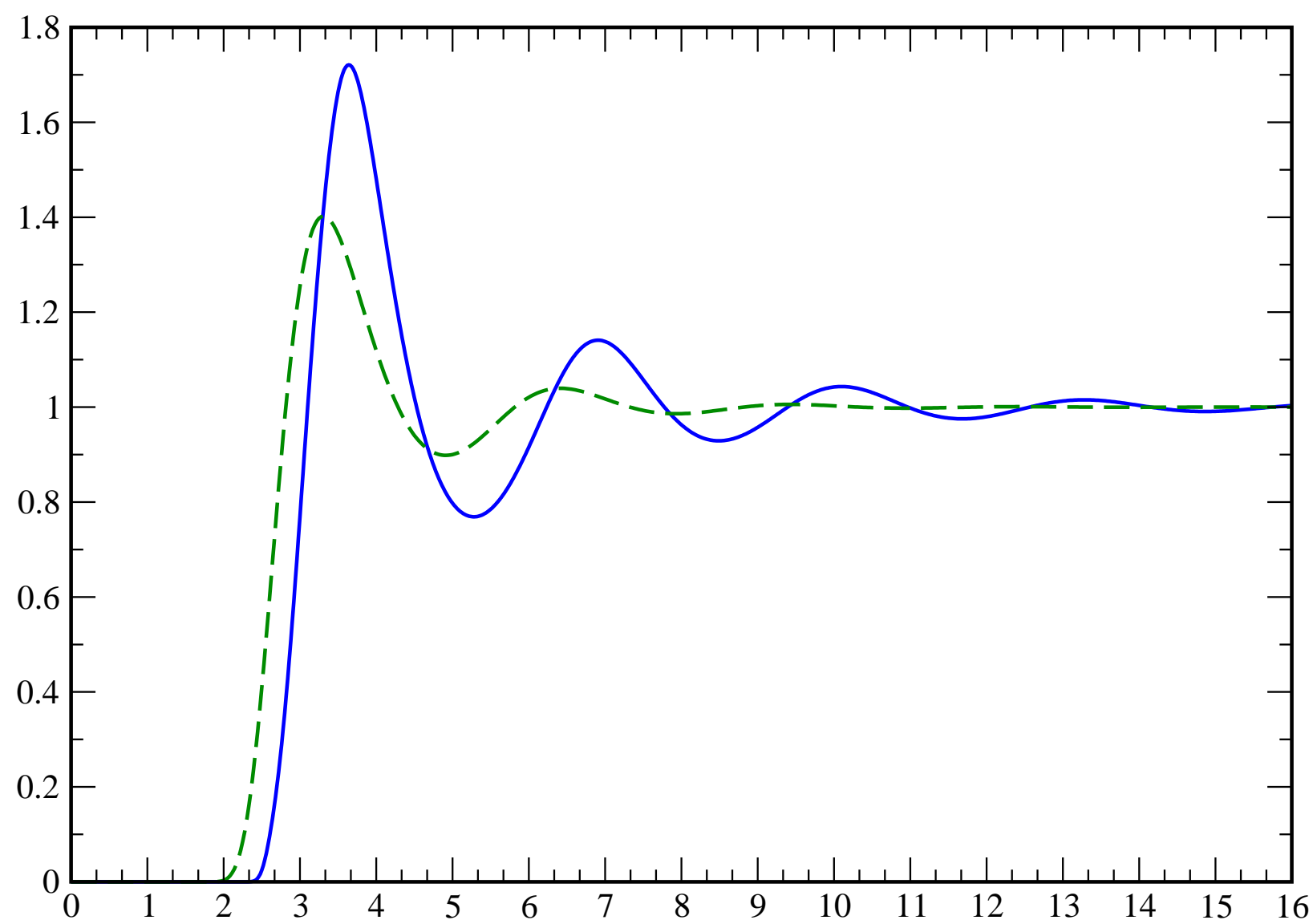

FIG. 2: Comparison of radial distribution functions of liquid para-hydrogen and of normal liquid helium (broken line) under similar thermodynamic conditions (abscissa: relative distance $r$ in units $[\AA])$. 
distribution $g(r)$ at large relative distances. The oscillations seen in the stochastic results at wavenumbers $k<1.5 \AA^{-1}$ are artifacts of the inherently unavoidable small box size used in the PIMC calculations. In contrast, CDM theory does not suffer from this deficiency since we can easily solve the Schrödinger equation in a sufficiently large interval of relative distances. CDM theory provides therefore an efficient tool for accurate calculations of the isothermal compressibility and isothermal velocity of sound in the limit of vanishing wavenumber.

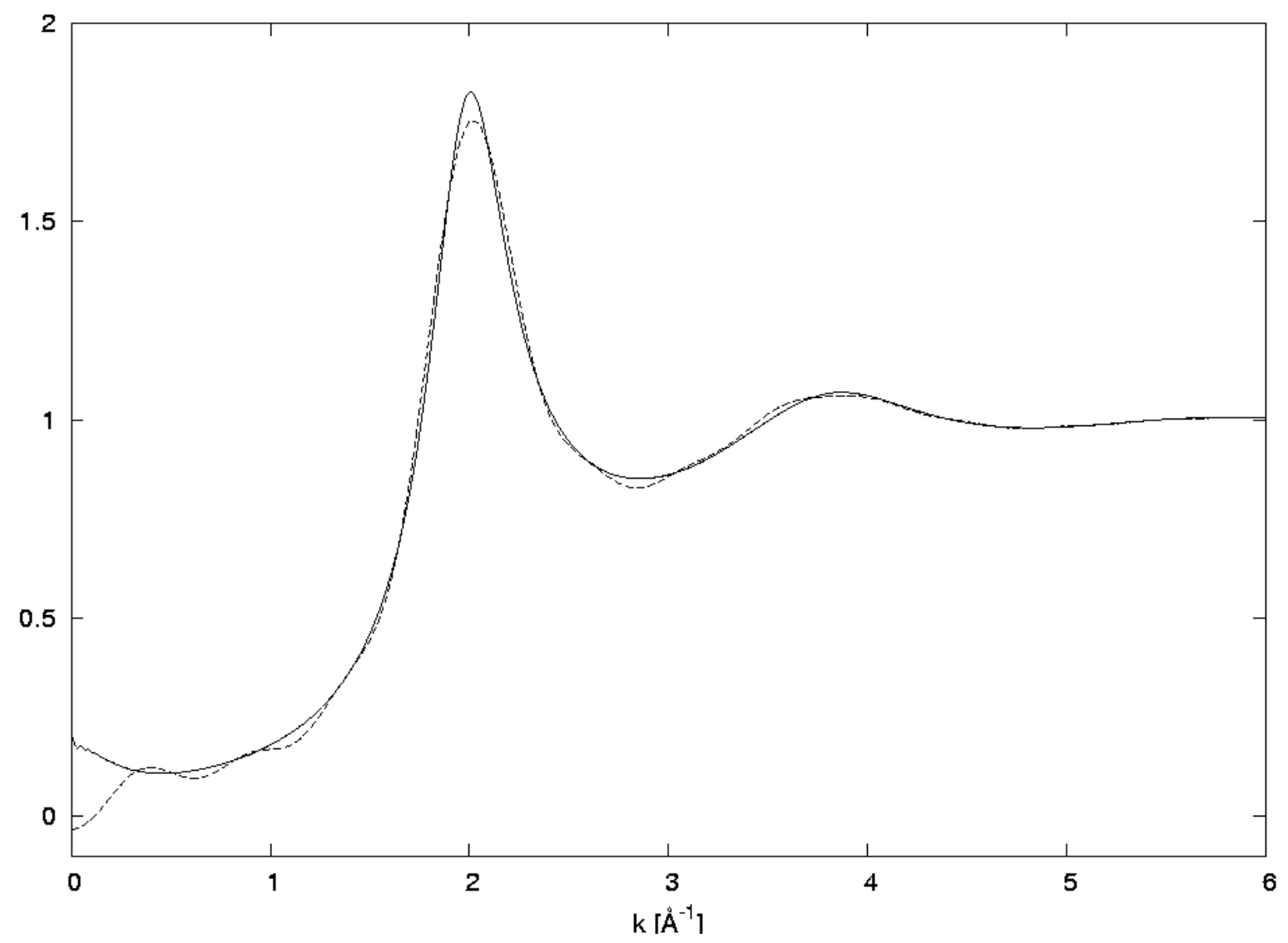

FIG. 3: Numerical results on the structure function $S(k)$ of liquid para-hydrogen by CDM theory and from PIMC calculations (dashed line), see text.

The theoretical results reported may be compared with experimental data from neutron scattering experiments ${ }^{7}$. The data processing yields an experimental structure function with a much higher first peak than found in the theoretical result on $S(k)$. This discrepancy between peak values of about 2.8 experimentally and about 1.8 for the theoretical result is not acceptable. There are theoretical data from molecular dynamics calculations ${ }^{\underline{\underline{T}}}$ which seem to support the experimental findings (see Figures 3 and 5 of Ref. 7). However, they are evidently 
in disagreement with the present results within CDM theory and of PIMC calculations. This problem -at least on the theoretical side- awaits a future careful examination.

\section{EXCITATIONS}

A correlated normal Bose fluid permits at least two different branches of excitations. There is a collective branch of phonons at small wavenumbers $\mathrm{k}$ and possibly roton-like excitations at atomic wavelengths. A second branch consists of excited quasiparticles that conserves the total number of constituents.CDM theory enables us to determine the excitation energies of both branches by solving two Euler-Lagrange equations ${ }^{12}$,

$$
\frac{\delta F_{\lambda}}{\delta n_{c c}(k)}=0
$$

with subcondition

$$
\sum_{\mathbf{k}} n_{c c}(k)=N
$$

and

$$
\frac{\delta F_{\lambda}}{\delta n_{\text {coll }}(k)}=0
$$

The thermodynamic potential $F_{\lambda}\left[S(k), n_{c c}(k), n_{\text {coll }}(k) ; T, \rho\right]$ is a functional of the static structure function $S(k)$, the momentum distribution $n_{c c}(k)$ of $N$ quasiparticles, and the occupation-number density $n_{\text {coll }}(k)$ of the collective excitations. An explicit expression for the functional $F_{\lambda}$ can be constructed within CDM theory by following a systematic approximation procedure. At the present level of formal development the functional is generated from a trial $N$-body density matrix of Jastrow- type. Future improvements may be derived by a suitable generalization of the Correlated Basis Functions (CBF) formalism for the ground state of a quantum many-body system ${ }^{18,19}$.

We have solved the Euler-Lagrange equations using the approximate thermodynamic potential derived from the Jastrow trial $N$-body density matrix ${ }^{12}$. At the assumed temperature $T=16 \mathrm{~K}$ and particle-number density $\rho=0.021 \AA^{-3}$ one finds that the optimal momentum distribution of the quasiparticles is excellently represented by the Gaussian form

$$
n_{c c}(k)=\exp \beta\left[\mu_{0}-\epsilon_{0}(k)\right]
$$

with $\epsilon_{0}(k)=\hbar^{2} / 2 m$ (molecular mass $\mathrm{m}$ of hydrogen) and inverse temperature $\beta=\left(k_{B} T\right)^{-1}$. The chemical potential is $\mu \simeq-7.8 \mathrm{~K}$ due to the subcondition (41). The coresponding 
one-body reduced density matrix is therefore given by

$$
n_{c c}(r)=\exp \left[-\pi\left(\frac{r}{\lambda}\right)^{2}\right]
$$

with a thermal wavelength $\lambda \simeq 3.09 \AA$. A consequence of this result is the absence of particle-exchange correlations in the hydrogen state considered, explicitly expressed by the result $11,12 S_{c c}(k)=G_{c c}(r) \simeq 0$. The quasiparticles are therefore distinguishable free hydrogen molecules obeying classical Boltzmann statistics.

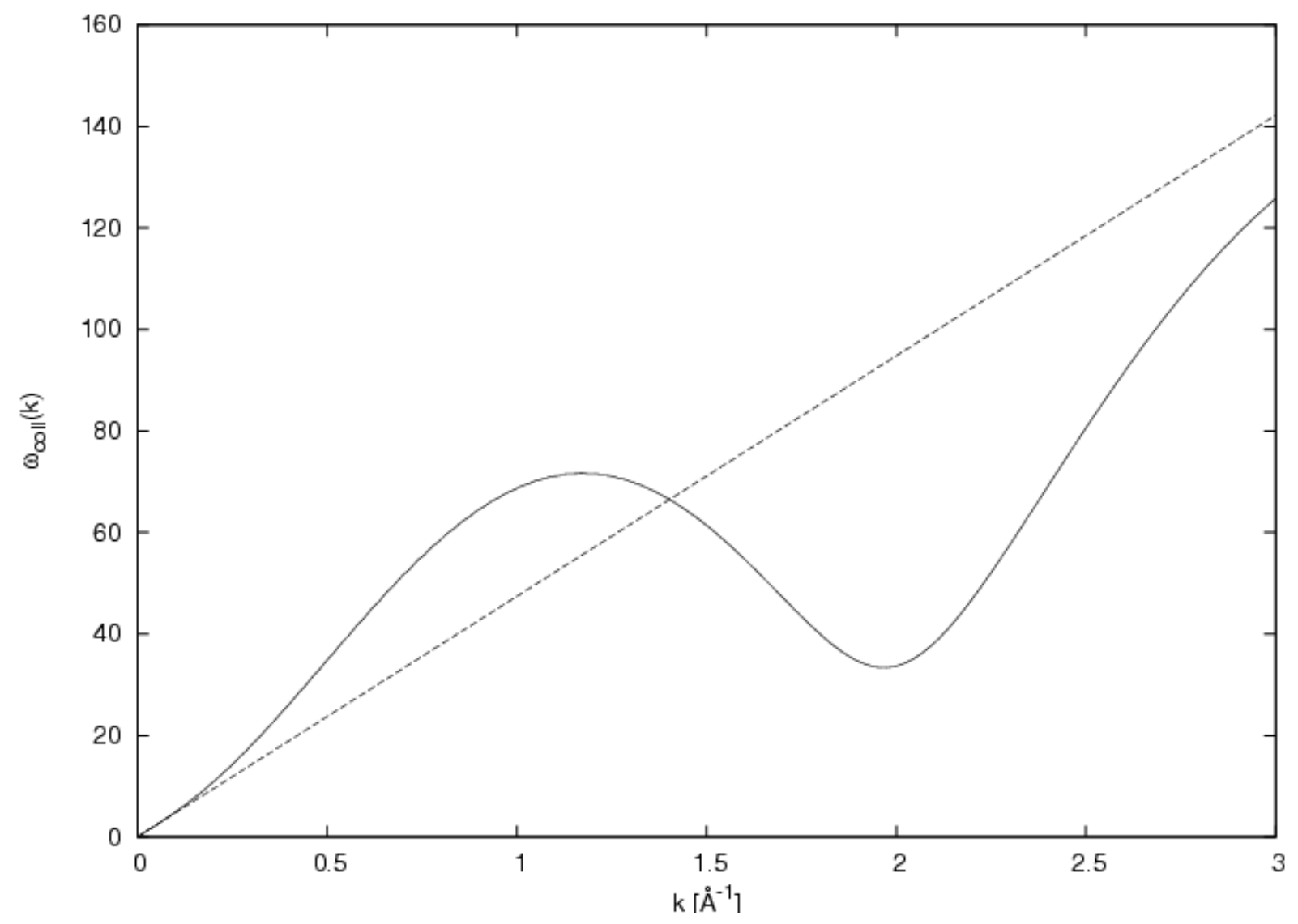

FIG. 4: Energy spectrum of collective excitations in the hydrogen system, in generalized Feynman approximation. The dashed line indicates the linear phonon spectrum in the limit of vanishing wavenumber.

Eq. (15) is a generalized Feynman equation ${ }^{12.20}$ that can be easily solved. The optimal excitation energies $\omega_{\text {coll }}(k)$ are displayed in Figure 4. This energy branch is of the familiar phonon/roton form. The broken line indicates the phonon limit $\omega_{\text {coll }}(k) \simeq \hbar c k$ as $k$ approaches zero. Its slope yields the theoretical isothermal sound velocity $c \simeq 680 \mathrm{~m} / \mathrm{s}$. 
We may compare these results with the available experimental data ${ }^{8}$ (cf. Figures 5 and 8 therein). There is good agreement between the theoretical and the experimental results in the roton region. However, the roton excitations are strongly damped.In contrast,the excitations in the wavenumber region $k<0.8 \AA^{-1}$ are stable, yet the theoretical excitation energies become significantly smaller than the experimental energies by increasing the wavenumber $k$. The discrepancy requires further investigation.

\section{MOMENTUM DISTRIBUTION}

The momentum distribution of a single hydrogen molecule in the liquid is given by the integral

$$
n(k)=\rho \int n(r) \mathrm{e}^{i \mathbf{k r}} d \mathbf{r}
$$

where function $n(r)$ is the (unit-normalized) one-body reduced density matrix. This quantity has been analyzed within CDM theory ${ }^{2.15}$. The formalism yields the structural factor decomposition

$$
n(r)=n_{c} N_{0}(r) \exp [-Q(r)]
$$

with the strength factor $n_{c}=\exp Q(0)$. The factor $N_{0}(r)=n_{c c}(r)+N_{c}(r)$ embodies the quantum-mechanical effects due to the exchange of identical particles. The function $P(r)=$ $Q(r) / Q(0)$ can be interpreted as the (unit-normalized) phase-phase correlation function.

Employing the hypernetted-chain (HNC) technique for evaluating the functions $\frac{15}{}$ we calculated $N_{0}(r)$ and $P(r)$ for liquid hydrogen in HNC/0 approximation. As expected, the exchange correlations $N_{c}(r)$ are very small and thus

$$
N_{0}(r) \simeq n_{c c}(r)
$$

Figure 5 displays theoretical results on function $\mathrm{P}(\mathrm{r})$ in $\mathrm{HNC} / 0$ approximation. We see that this distribution is well represented by a Gaussian form

$$
P(r) \simeq P_{G}(r)=\exp \left[-\pi\left(\frac{r}{\lambda_{P}}\right)^{2}\right],
$$

with $\lambda_{P} \simeq 3.73 \AA$ at $T=16 \mathrm{~K}$ and $\rho=0.021 \AA^{-3}$. The strength factor $n_{c}$ is related to the curvature of function $P(r)$ and the total kinetic energy per molecule of the Bose liquid. A 


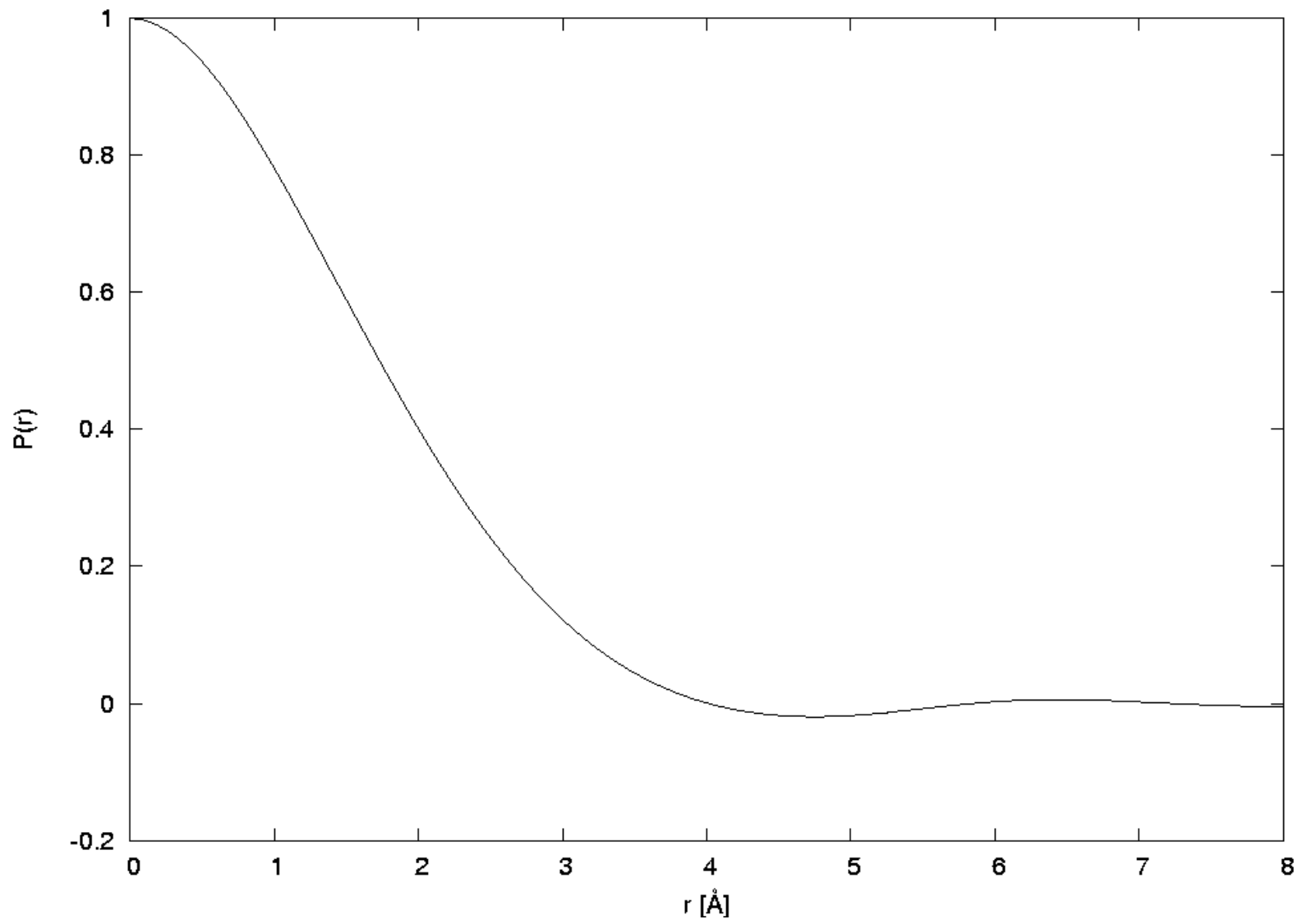

FIG. 5: Numerical results for the phase-phase correlation function $P(r)$ by CDM theory, in HNC/0 approximation.

PIMC calculation of this energy portion yields $58.6 \mathrm{~K}$ at $T=16 \mathrm{~K}$ and $\rho=0.021 \AA^{-3}$. With these input data the strength factor has the value $n_{c}=0.118$.

Straightforward multiplication and integration via Eq. (99) yield the one-body elements $n(r)$ and the momentum distribution $n(k)$. Figure 6 represents numerical results on the dependence of the latter function on wavenumber $k$. We may compare it with the Gaussian momentum distribution that would hold by assuming that the molecules are free and distinguishable but have the same total kinetic energy per particle as the quantum liquid. This distribution would be characterized by an effective thermal wavelength $\lambda_{0} \simeq 1.97 \AA$ (Figure 6 , broken line). Obviously, the difference

$$
\Delta N(k)=k^{2}\left[n(k)-n_{G}(k)\right]
$$

measures the deviation of the kinetic energy distribution of the quantum liquid from the classical Maxwell distribution of distinguishable hydrogen molecules. Its dependence on 


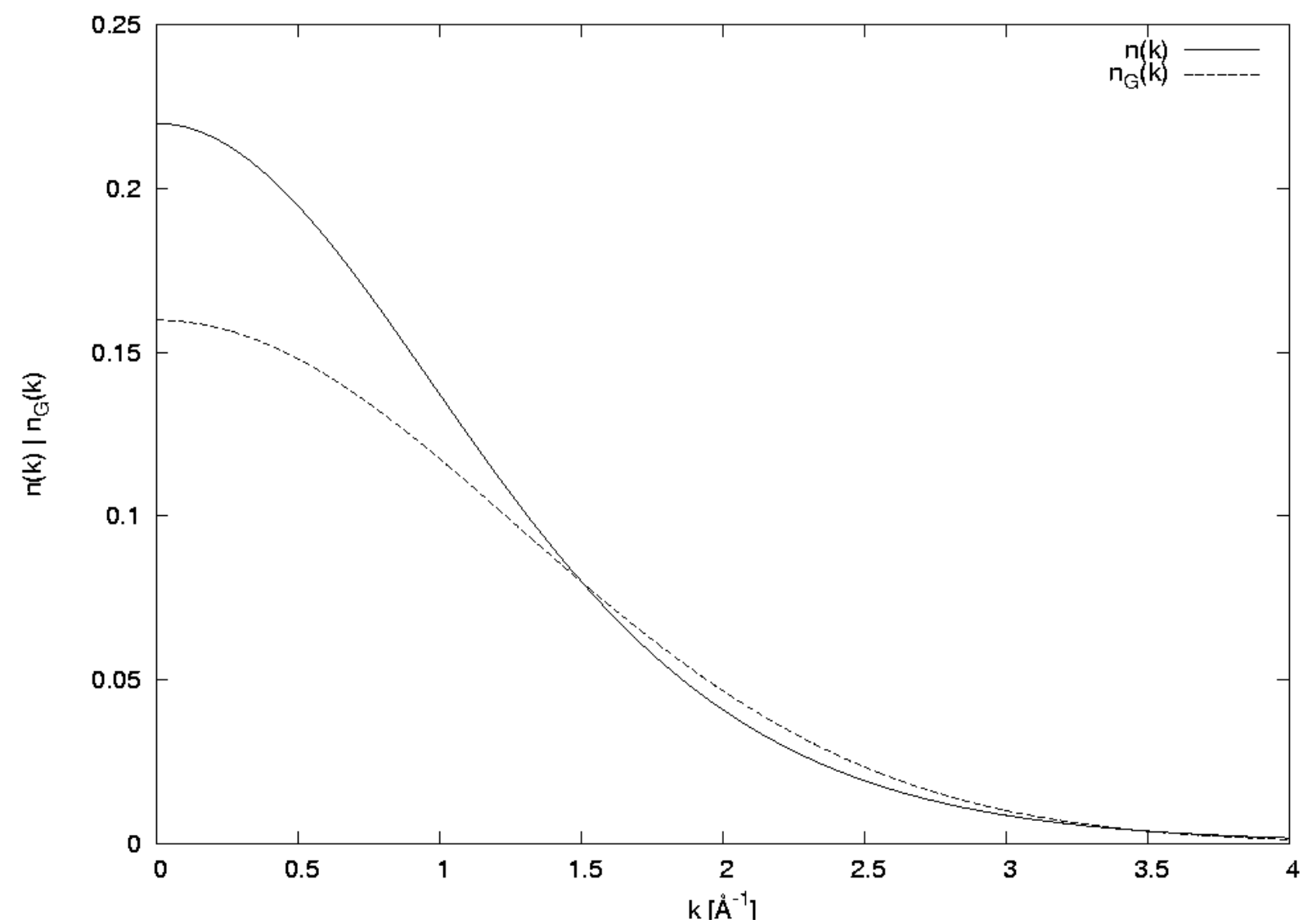

FIG. 6: Theoretical momentum distribution $n(k)$ of a single hydrogen molecule in the liquid. It is compared to the classical Gauss distribution $n_{G}(k)$ that leads to the same total kinetic energy per molecule as the correlated hydrogen liquid ( broken line).

wavenumber $k$ is displayed in Figure 7. We see that the quantum-mechanical energy distribution of the system favors an increase of the number of molecules with low momenta compared to the classical distribution.

\section{SUMMARY}

We have presented a theoretical and an experimental analysis of the structure of liquid para-hydrogen close to the triple point. The system is characterized by very strong dynamical spatial correlations induced by the intermolecular forces. However, the repulsion at short relative distances suppresses particle-exchange correlations between and among the 


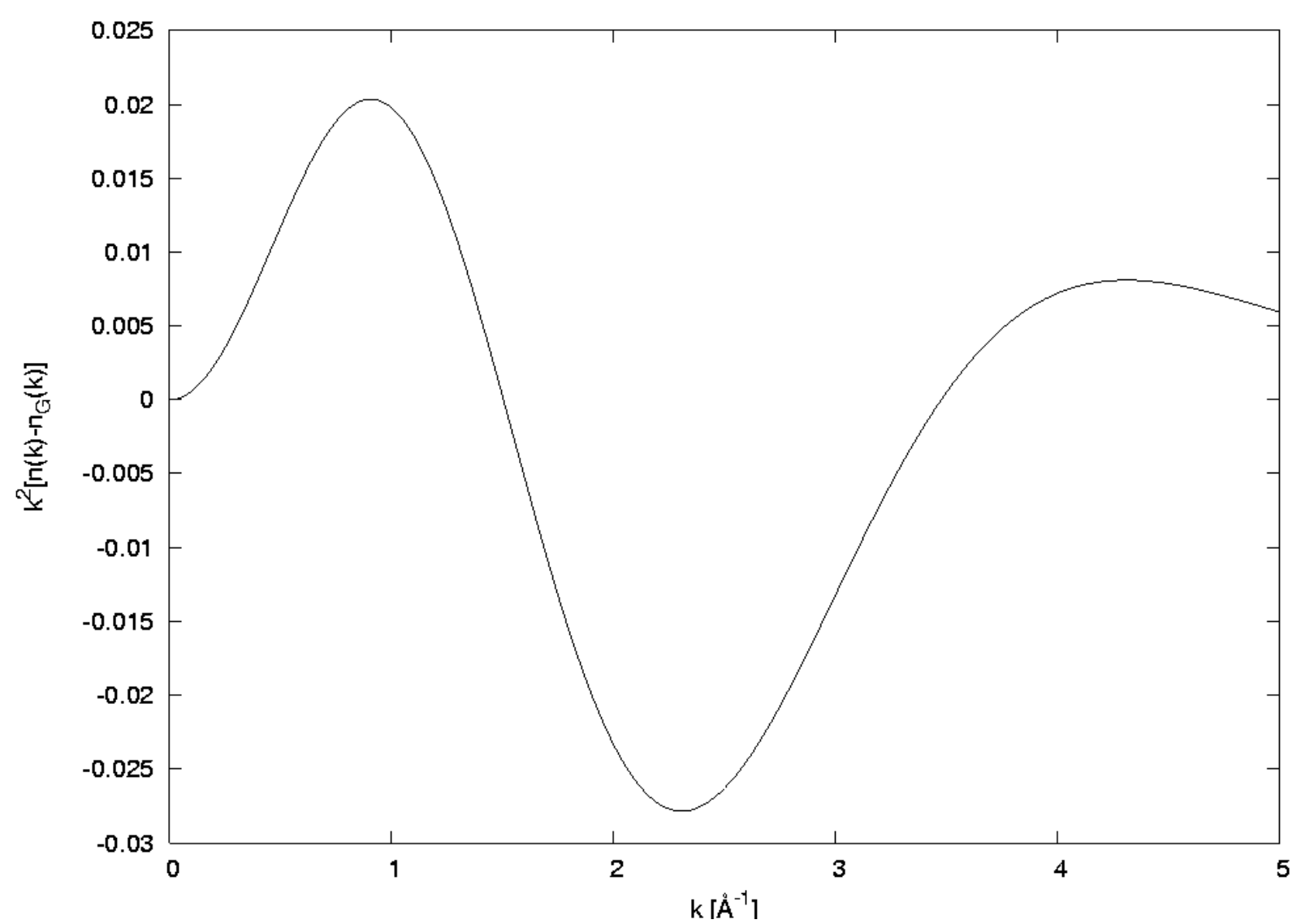

FIG. 7: Deviation of the kinetic energy distribution $k^{2} n(k)$ of liquid para-hydrogen from the corresponding classical Maxwell energy distribution (cf. Eq. (12)).

hydrogen molecules. For the same reason, the exchange (or cyclic) correlation function and the associated exchange structure function are almost zero everywhere. Furthermore, the quasiparticle momentum distribution is very well approximated by the classical Gaussian distribution of free and distinguishable particles. Similarly,the short-ranged phase-phase correlation function and corresponding structure function are to a very good approximation of Gaussian form. The interplay of exchange and phase-phase coupling, however, generates a significant departure of the single-particle momentum distribution in liquid para-hydrogen from the classical Maxwell-Gauss distribution.

The present study employed the formalism of CDM theory for a normal Bose fluid. The results have been compared with PIMC simulation data. The numerical comparison demonstrates the high accuracy of CDM theory when applied to liquid hydrogen at low temperatures. CDM theory is therefore expected to be a very fast, efficient, and reliable 
tool for a detailed quantitative analysis of normal Bose fluids under similar thermodynamic conditions.

1 K. A. Gernoth and M. L. Ristig, in Particle Scattering, X-Ray Diffraction, and Microstructure of Solids and Liquids,Lecture Notes in Physics 610, eds. M. L. Ristig and K. A. Gernoth,(SpringerVerlag, Heidelberg, 2003).

2 P. E. Sokol, R. N. Silver, and J. W. Clark, inMomentum Distributions, eds. R. N. Silver and P. E. Sokol,(Plenum, New York, 1989).

3 D. G. Fried, T. C. Killian, L. Willmann, D. Landhuis, S. C. Moss, D. Kleppner, and T. J. Greytak, Phys. Rev. Lett. 81, 3811 (1998).

4 E. A. Cornell and C. E. Wieman, Rev. Mod. Phys. 74, 875 (2002).

5 M. Ross, Rep. Prog. Phys. 48, 1 (1985).

6 S. Bonev, E. Schwegler, T. Ogitsu, G. Galli, Nature 431, 669 (2004).

7 J. Dawidowski, F. J. Bermejo, M. L. Ristig, B. Fak, C. Cabrillo, R. Fernandez-Perea, K. Kinugawa, and J. Campo, Phys. Rev. B 69, 014207 (2004).

8 F. J. Bermejo, B. Fak, S. M. Bennington, R. Fernandez-Perea, C. Cabrillo, J. Dawidowski, R. Fernandez-Diaz, P. Verkerk, Phys. Rev. B 6015154 (1999).

9 J. Dawidowski, F. J. Bermejo, M. L. Ristig, C. Cabrillo, and S. M. Bennington, Phys. Rev. B (submitted).

10 G. Senger, M. L. Ristig, K. E. Kuerten, and C. E. Campbell, Phys. Rev. B 33, 7562 (1986).

11 G. Senger, M. L. Ristig, C. E. Campbell, and J. W. Clark, Ann. Phys. (N.Y.)218, 160 (1992).

12 T. Lindenau, M. L. Ristig, J. W. Clark, and K. A. Gernoth, J. Low Temp. Phys. 129, 143 (2002).

13 K. A. Gernoth, Ann. Phys. (N.Y.) 291, 202 (2001).

14 I. Silvera and V. Goldman, J. Chem. Phys. 69, 4209 (1978).

15 R. Pantfoerder, T. Lindenau, and M. L. Ristig, J. Low Temp. Phys. 108, 245 (1997).

16 K. A. Gernoth, M. J. Harrison, and M. L. Ristig, these Proceedings.

17 R. A. Aziz, V. P. S. Nain, J. S. Carley, W. L. Taylor, and G. T. McConville, J. Chem. Phys. 704330 (1979).

18 M. Saarela, Phys. Rev. B 33, 4596 (1986). 
19 E. Krotscheck, in Microscopic Quantum Many-Body Theories and Their Applications, Lecture Notes in Physics 510, eds. J. Navarro and A. Polls (Springer-Verlag, Berlin, Heidelberg 1998).

20 R. P. Feynman, Phys. Rev. 94, 262 (1954). 